\title{
Correlations between Logistics and Infrastructure Digitalization and Gross Domestic Product Size
}

\author{
Natalia Alekseeva \\ Institute of Industrial Management \\ Economics and Trade \\ Peter the Great St.Petersburg \\ Polytechnic University (SPbPU) \\ St. Petersburg, Russia
}

\author{
Larisa Voronova \\ Graduate school of foreign languages, \\ Institute of Humanities \\ Peter the Great St.Petersburg \\ Polytechnic University (SPbPU) \\ St. Petersburg, Russia
}

\author{
Nadezhda Grashchenko \\ Institute of Industrial Management, \\ Economics and Trade \\ Peter the Great St.Petersburg \\ Polytechnic University (SPbPU) \\ St. Petersbrg, Russia
}

\begin{abstract}
The life of a contemporary person acquires a new quality due to smart solutions, hence the issue of what affects the appearance of smart solutions and what impact smart solutions have on economic activity indicators is relevant. The paper is aimed at identifying correlations between urban logistics and infrastructure digitalization and a Gross Domestic Product size. This paper explores the most popular digital solutions that affect the quality of logistics and infrastructure. This paper shows that a rather significant correlation is observed between the logistics and infrastructure digitization indices and the Gross Domestic Product indices adjusted for per capita purchasing power parity. This paper demonstrates that the higher logistics and infrastructure digitization contributes to the cost rising of domesticmanufactured products. Conversely, the higher Gross Domestic Product level raises the possibility to create the more advanced and high-quality digital logistics and infrastructure. The authors concluded that the identified correlation is missing in countries having the highest gross domestic product indices adjusted for per capita purchasing power parity and their indices of digital logistics and infrastructure development are at a medium level.
\end{abstract}

Keywords-digitalization, digital technologies, logistics, infrastructure, smart applications

\section{INTRODUCTION}

Today, the human life acquires a new quality due to smart solutions. Through the use of technology and the digitalization of traditional services, people use their resources and time more rationally and productively becoming residents of smart cities [1].

The Smart City concept formed in the early 2000s was aimed primarily for the development of technologies and infrastructure [2]. New technologies, large data processing centres, smart sensors and automated power grids have emerged as a result of further research and investment [3] greatly influencing the quality of life in cities, traffic convenience, safety, ecology and power consumption [4]. McKinsey experts point [5] out that the world cities differ greatly in the number of implemented groups of digital solutions, but all of them give due consideration to transport systems [6].

Among the digital solutions that affect the quality of logistics and infrastructure the following three are the most notable: smart parking, car sharing, smart traffic management on highways.

\section{A. Smart Parking}

Using Smart Parking applications, drivers can search for free parking spaces nearby and pay for them [7]. Research results [5] confirm the current digitalization of all stages of a parking process - from searching for a free space to car parking and payment making. The main two functions are used in many cities: navigation to a parking place and making an electronic payment for parking via a smartphone application. Utilising applications from third-party developers, users can also reserve parking spaces and make prepayments in many cities where the authorities cooperate with parking lots and covered parkings. Moscow (A1 PARK), New York (ParkMobile) and Singapore (Park\&Go) can be cited as good examples.

Most applications for parking in cities appeared only 3-5 years ago, and there is still the considerable potential for improving their functionality [8]. In order to avoid unnecessary transport movements in the process of searching for a free parking space, the navigation solution to track unoccupied places must be developed. Although the existing parking occupancy monitoring technologies have some limitations, it is possible to search for additional opportunities by combining data from several sources and this often requires interaction between the state and the private sector. Closed Circuit Television (CCTV) cameras and optical / magnetic position sensors transmit data about occupied parking spaces, which are then processed and transmitted to a user interface. However, this technology may have certain restrictions such as high costs and the need to install infrastructure facilities to provide communication between sensors and repeaters as well as between repeaters and servers. Moreover, the sensors must be installed into asphalt or concrete, which is also fraught with difficulties. Additionally, some attempts to implement this model ended in failure due to the fact that the street sensors had not functioned properly in the areas, which were exposed to adverse weather conditions (dirt, dust, ice and water can affect the sensor readings). Nevertheless, the cities with the largest implementation of Smart Parking solutions (for example, Shanghai, Hong Kong, Singapore and Barcelona) use such sensors. Using the geolocation data and signals from the navigation application accelerometer, one may find recently vacated parking spaces and forecast the percentage of free places in a particular area, and using the vehicle traffic data, it may be possible to calculate the percentage of free parking spaces in the area during the given time period. 
Although this technology is already used in a number of applications, not all the navigation solutions use it. The limitations of this technology are attributed to the fact that drivers use different navigation systems (and some do not use them at all), and therefore the forecast is not always accurate. Applications for a parking payment collect and send data about users leaving their car after parking, and sometimes they can even predict how long the driver will be absent. Since the parking lots are often occupied by local residents, the application does not always allow predicting whether the parking space will be available. Local residents can be exempted from the responsibility to register in the system if they are allowed to park in a particular zone. Mobile vans equipped with a video surveillance system photograph cars and provide information about free parking spaces and illegally parked cars. Such information about occupied parking spaces in the system is not always relevant, since it is updated only several times per hour. The next generation solution for the city parking can rely on a dynamic pricing pattern so that the city authorities could regulate the demand for parking spaces. To implement this it is necessary to increase the parking rates at certain hours in congested areas, which will lead to the possibility to find at least few free parking spaces at any time there.

\section{B. Car Sharing}

One of the popular solutions for the logistics and infrastructure digitalization domain is the use of Car Sharing, which is a short-term car rental. Parkring-based Car Sharing has existed for a long time. In some large cities this service appeared 15-20 years ago. A new rise of Car Sharing popularity began 3-7 years ago, when the models without being tied to parking lots or specially reserved parking spaces appeared. Several examples confirm that the spread of Car Sharing can contribute to the increase of public transport in the passenger traffic structure [9]. According to the survey conducted in Switzerland, the emergence of Car Sharing led to the decrease in private car trips by $35 \%$, and the frequency of public transport use among residents participating in Car Sharing programs increased by $12 \%$ [5]. The survey conducted in France showed that users of Car Sharing services drove private cars less frequently and used public transport more often [10].

The population density affects the demand for Car Sharing services, since in large cities it is difficult to keep cars in close proximity to users. Government regulation at a city level (for example, the availability of free parking spaces for Car Sharing in European cities), restriction measures against online taxis and high taxes for car owners can also contribute to the spread of Car Sharing. And yet there are a number of limitations for the current Car Sharing model distribution. According to the experts' opinion, the Car Sharing business model is realizable in the cities having the population of over 500000 citizens, since the high utilization ratio is required to maintain competitive prices [5]. At a city level, it is possible to achieve significant positive results due to its large scale. All other things being equal, the higher degree of the urban market consolidation (fewer suppliers) contributes to the increase in the car utilization factor and profitability of the relevant services [11].

\section{Smart Traffic Management on Highways}

Traffic safety depends directly on the awareness of city and road services and drivers about the situation on the roads in the real-time mode [12]. Traffic management systems based on CCTV and the use of business intelligence technologies are designed to solve this issue [13]. Recently, CCTV systems have become an integral part of the integrated security system, since modern controllers allow not only monitoring and recording video, but also controlling the security system response in case of unforeseen situations [14]. Particularly, the traffic management systems can analyze the road traffic, recognize different types of vehicles, determine license plates, car colour, and compare them with those in the hijacked car database, for instance [15].

The situation in London can be given as an example of smart traffic management on highways. The London Traffic Control Centre is a special service which is responsible for the city road traffic situation. It monitors the car movement via more than 1000 surveillance systems, adjusts the time of traffic lights switching, calls the traffic services when an accident happens, and displays traffic recommendations or latest updates on special indicator boards. The data about a road repair or closure in the real time can be obtained from the Internet. One can also plan a trip schedule interactively by entering the starting and ending points and the system will automatically choose the best route. In Moscow, the Start system enables the monitoring of the vehicle traffic almost throughout the entire city centre. It includes the control centre, video monitor systems, traffic lights, CCTV cameras, traffic information boards, controllable road signs, etc. Having received the information from the radar and television sensors installed on the streets and having processed it, the Start computer centre sends signals to controllable speed indicators [16]. Using this system, the situation on the city main highways and road interchanges is monitored from the single dispatch centre and prompt actions are being taken to regulate the traffic in one or another part of the capital. Currently, more than 120 main transport hubs and intersections in the Moscow city centre are monitored using the teleautomatic Start system.

\section{Correlations between the urban logistics and infrastructure digitalization and the GDP size}

The search for the correlations between the GDP size and the indices of the world countries' economic activity development and their position has been occupying the minds of scientists for a long time. In [17], the correlation of indices of the financial sector development and the GDP per capita in the countries of the European Union is investigated. Another study [18] explores the effect of direct foreign investment on the employment and the GDP in India. The author also refers to the work of Hooda [19], which analyzes the impact of direct foreign investment on the Indian economy using the GDP index. Cox et al. [20] show that the GDP indices are higher in the countries where freedom of expression and individual initiative prevails. This study also demonstrates that the better the relationship between the society and the government is organized, the higher the GDP indices are. In addition, it notes that the national wealth growth can be increased by the society's orientation on the issues of quality of life, greater employment opportunities, greater accessibility of goods and services and more 
advanced education systems. Cox et al. also draw attention to the correlation between the environmental protection and the GDP and show that the higher the GDP is the more consideration is given to the environmental issues.

The development of digital economy has prompted researchers to consider aspects of its influence and dependence on the welfare of the country. The most common opinion is that the digitalization of economy has a positive effect on the productivity and economic growth. For instance, the studies [21, 22] indicate that the use and distribution of broadband communication stimulates the productivity and economic growth. The surveys [23, 24] show that the labour productivity increases with the investments in the computerization of business processes and the development of the Internet network. The survey [25] demonstrates that the countries' well-being grows along with the digitalization index while exhibiting increasing returns to scale. The work graphically illustrates the dependence of the digitalization index on the GDP per capita and vice versa.

Thus, one can say that studies on the correlations between the GDP and indices characterizing the development of digital economy are relevant. Although, the researchers consider that the issues of the urban logistics and infrastructure digitalization remain insufficiently studied despite the fact that there are a number of publications devoted to the analysis of the use of car sharing, smart parking, and smart traffic management on highways [5, 7$16]$.

McKinsey experts point out [5] that the level of using digital technologies depends on both the citizens' desire and activity in changing their habitual behaviour [26] and the city authorities, whose role may vary from the restrictive regulation to the official support and implementation management. In our opinion, the Gross Domestic Product (GDP) index is also formed and depends on the business activity wherein people work, their desire to change due to market competition and the government structures that help or hinder business development. Therefore, the question, whether there is a correlation between indices indicated in the title of this article, is of utmost interest to the researchers.

The MGI study "Smart Cities: Digital Solutions for a More Livable Future" showed that the cities with high income typically have high-speed data networks and extensive sensor systems [27]. In this study, the researchers assume that there may be a correlation between the GDP size and the level of logistics and infrastructure digitalization. Therefore, this paper aims to identify the correlation between the logistics and infrastructure digalization and the GDP size.

\section{MATERIALS AND METHODS}

In this paper the researchers have proposed the hypothesis that the level of logistics and infrastructure digitalization is interrelated with the GDP of the country wherein they are implemented.

The logistics and infrastructure digitalization indices are considered using the index mean value according to the following parameters:

- The use of smart parking applications (hereinafter referred to as "Smart Parking").
- The number of cars in Car Sharing in relation to the city population (hereinafter referred to as "Car Sharing").

- The level of smart traffic management on highways (hereinafter referred to as "Traffic").

McKinsey analysts discovered that due to the different climatic situation in different countries and world cities, some urban dwellers do not need the developed bicycle infrastructure and its rental. Since our research explores all world countries, the bicycle infrastructure digitalization indices have not been taken into consideration.

The indices in the study were taken from the EasyPark Group report [28]. EasyPark Group analysts assessed 500 world cities. In our research the urban indicators were aggregated to obtain the country indices. The mean value for each city included in the EasyPark Group report was calculated for each country.

The peculiarity of the EasyPark Group sampling lies in that the analysis took into account only countries with very high and medium development levels determined by the human development index and considered the cities included in the UN prosperity list and the European City Digital Index. This sampling did not include new cities that were intended to be designed as the smartest in the world but not yet fully completed or not widely known (for example, the city of Masdar) due to lack of data. Each parameter in the EasyPark Group sample was estimated from 1 to 10 ; the higher the score the better the effectiveness of digital technologies implemented and used in the city.

The use of Smart Parking applications was influenced by the combination of the following elements: the percentage of citizens who own cars, the number of parking spaces per 1 square $\mathrm{km}$ in the city centre, the use of smartphones and the availability of parking applications. The level of smart traffic management on highways was determined based on the TomTom traffic index and the INRIX traffic scorecard.

The selected Parking, Car Sharing and Traffic indicators were evaluated for multicollinearity, as a result only the Smart Parking and Traffic indices were used in the calculations to identify the correlation between the urban logistics and infrastructure digitalization and the GDP size.

Since the EasyPark Group compiled the report as of 2017, the GDP indices for 2017 were used to observe the compliance principle. As this paper compares the urban logistics and infrastructure digitalization around the world, having considered that the consumer basket price varies in different countries, the researchers used the GDP index adjusted for the purchasing power parity. Given the fact that world countries differ in size, this index was adjusted for per capita. The value of the selected index was determined based on the International Monetary Fund data sources.

The analysis of studies to identify the correlations between the GDP and the selected analyzed index showed that the graphical method was a widely applied research method [25], thus it was utilised in this research. In the study [17] the determination coefficient was used to substantiate the relationship. The study [20] was performed based on the analysis of the correlation coefficient between the GDP 
index per capita and the cultural aspect index as well as on the analysis of the correlation coefficient between the environmental sustainability index and the GDP per capita. Therefore, the correlation and determination coefficients were used in this research to determine the relationship. Additionally, the Student t-test and the Pearson coefficient were used to determine the relationship between the variables under consideration.

\section{RESULTS}

The selected values of logistics and infrastructure digitalization indices have been checked for collinearity (see Table 1)

TABLE I. CORRELATION RATIOS FOR THE SELECTED INDICES

\begin{tabular}{|l|c|c|c|}
\hline \multicolumn{1}{|c|}{ Factor } & Smart Parking & Car Sharing & Traffic \\
\hline Smart Parking & 1.00 & 0.48 & 0.23 \\
\hline Car Sharing & 0.48 & 1.00 & -0.04 \\
\hline Traffic & 0.23 & -0.04 & 1.00 \\
\hline
\end{tabular}

The obtained data in the Table 1 demonstrate the weak or nearly zero relationship between the selected indices. However, a more detailed examination of collinearity between the "Car Sharing" and "Smart Parking" indices shows that the correlation between these indices is missing only in Hungary, India, Taiwan and South Korea. If we remove the abovementioned countries from the sampling, the correlation value between the "Car Sharing" and "Smart Parking" indices reaches 0.70 , which can be characterized as a strong correlation. An additional analysis has identified the collinearity between the "Car Sharing" and "Smart Parking" indices. Therefore, it has been decided to exclude the "Car Sharing" index from the further analysis. Thus, the index assessing the logistics and infrastructure digitalization level has been obtained by multiplying the "Smart Parking" and "Traffic" indices.

The Figure 1 contains the graph evaluating the index correlation between the logistics and infrastructure digitalization level and the GDP size.

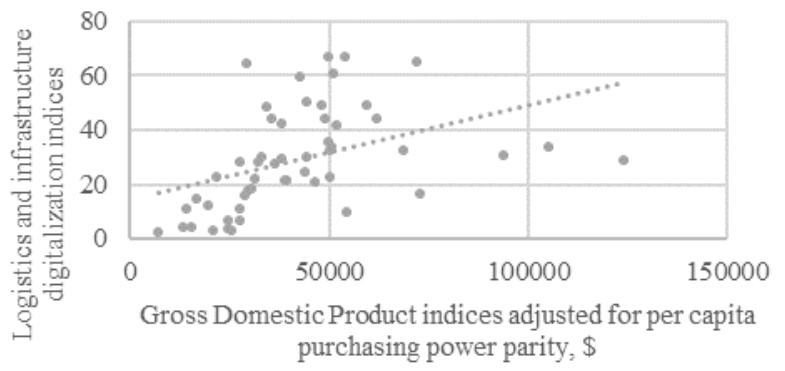

Fig. 1. Correlations between the logistics and infrastructure digitalization and the GDP size for 52 countries.

The graph in the Figure 1 illustrates that the level of logistics and infrastructure digitalization in the countries having one of the highest GDPs per capita is not as high. These countries include Saudi Arabia, the United Arab Emirates, Ireland, Singapore, Luxembourg and Qatar. Conversely, the situation in Hungary is quite the opposite; the digitization level is rather substantial considering the low level of GDP per capita. The data on the abovementioned countries have been excluded from the sample as outliers. As a result, the graph given above transformed into the one shown in the Figure 2.

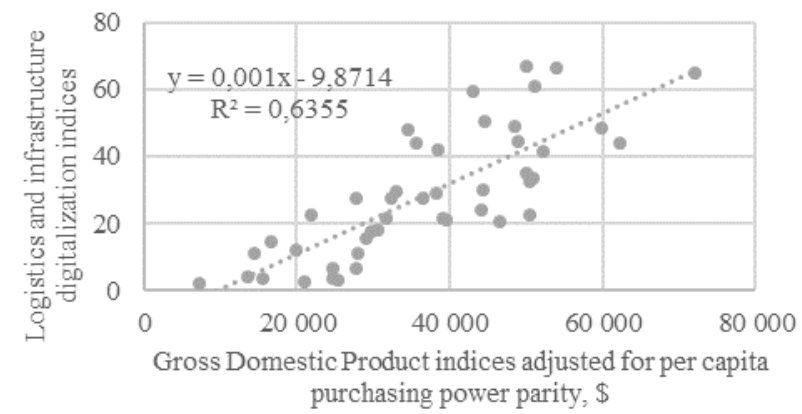

Fig. 2. Correlations between the logistics and infrastructure digitalization and the GDP size for 45 countries.

The relationship between the logistics and infrastructure digitalization and the GDP size for 45 countries is described by the correlation coefficient of 0.7 , which characterizes this correlation as strong. When using the linear approximation, the determination factor is equal to 0.64 (Figure 2). The number of degrees of freedom is 43 . The Student's t-value equals 8.647 with the critical value of 2.018 for a given number of degrees of freedom, which determines that the correlation is statistically significant. The Pearson coefficient is 0.8 , which determines the relationship as strong.

\section{DISCUSSION}

As can be seen from the graphs, the significant correlation is observed between the logistics and infrastructure digitization indices and the GDP indices adjusted for per capita purchasing power parity. The correlation value is 0.7 and the determination value is 0.64 .

Thus, it can be said that the higher logistics and infrastructure digitization contributes to the raise of domestic-manufactured products cost. Similar trends were identified in [21-24] based on the example of the development of computerization and broadband communication. This might occur due to the improvement of people's quality of life, reduction of the amount of time that labour resources need to commute or the location of contractors, creation of conditions for the use of employees' intellectual potential. Proper city logistics reduces the amount of effort that employees make to commute. The released forces can be used for work or rest, which is also necessary for the effective work.

Conversely; the higher GDP level provides the opportunity to create a more advanced and high-quality digital logistics and infrastructure. The increase in the orientation of the society towards the issues of quality of life with the GDP growth was also noted in [20]. However, this pattern is not always implemented in practice. Qatar and Luxembourg can be given as an example. These countries have the highest GDP adjusted for per capita purchasing power parity, 124,112 and 105,147 respectively, although the logistics and infrastructure digitalization index is 4.35 in Qatar and 5.33 in Luxembourg. Thus, one can see that the richest countries in the world keep the urban logistics and infrastructure digitalization at a medium level. Such 
countries, where the GDP per capita is high and the logistics and infrastructure digitalization level is at a medium level, also include Saudi Arabia, the United Arab Emirates, Ireland and Singapore.

The situation in Hungary is quite the opposite; the digitization level is substantial, while the level of GDP per capita is low. At the same time, Hungary was referred to the list of countries with no correlation between "Car Sharing" and "Smart Parking" indices. The reason lies in that the Car Sharing market in Budapest began to develop relatively recently, in 2018, which had not been reflected in the data used for the research. Smart Car Parking was actively developing in Budapest instead of Car Sharing, which led to the situation presented in the Figure 1 indicating that the level of digitalization turned out to be quite substantial at a low level of the GDP per capita.

\section{CONCLUSIONS}

The conducted study shows that the significant correlation is observed between the logistics and infrastructure digitization indices and the GDP indices adjusted for per capita purchasing power parity. The higher logistics and infrastructure digitalization contributes to the raise of domestic-manufactured products cost. In contrast, the higher GDP level provides the opportunity to create the more advanced and high-quality digital logistics and infrastructure. However, the correlation is missing in the countries having the highest GDP indices adjusted for per capita purchasing power parity, while their development indices of digital logistics and infrastructure are at a medium level.

The further research requires the presented analysis applied in the context of the Gross Regional Product generated in the cities under study. The indices that determine the use of digital technologies in logistics and infrastructure can be expanded, for instance, taking into consideration the level of collection charges for the use of Car Sharing as compared with the public transport charges.

\section{REFERENCES}

[1] A. Lepekhin, A. Borremans, O. Iliashenko, "Design and implementation of IT services as part of the "smart City" concept," MATEC Web of Conf. Int. Sc. Conf. on Bus. Tech. for Sust. Urb. Dev., SPbWOSCE Russian Federation, vol. 170, no. 01029, June 2018. DOI: $10.1051 /$ matecconf/201817001029.

[2] E. Ismagilova, L. Hughes, Y.K. Dwivedi, K.R. Raman, "Smart cities Advances in research-An information systems perspective," International Journal of Information Management, 2019, vol. 47, pp. 88-100.

[3] D.J. Bunders, K. Varr, "Problematizing data-driven urban practices Insights from five Dutch 'smart cities'," Cities, 2019, vol. 93, pp. 145152 .

[4] N. Alekseeva, N. Antoshkova, S. Pupentsova, "Application of the Monte-Carlo Simulation Method in Building and Energy Management Systems," Advances in Intelligent Systems and Computing. Russian Federation, vol. 983, pp. 257-266, December 2018. DOI: 10.1007/978-3-030-19868-8_26.

[5] J. Jonathan, E. Kuznetsova, "Smart technologies cities: what influences the choice of the citizens?," July 2018, unpublished (in rus).

[6] A.I. Levina, A.S. Dubgorn, O.Y. Iliashenko, "Internet of things within the service architecture of intelligent transport systems," EECS 2017 Eur. Conf. on Elect. Eng. and Comp. Sc. Switzerland, vol. 1, pp. 351 355, July 2018. DOI: 10.1109/EECS.2017.72.
[7] S. Evseeva, O. Kalchenko, O. Evseeva, "Innovative projects for sustainable development of cities (case of Saint-Petersburg)," MATEC Web of Conf. Int. Sc. Conf. on Bus. Tech. for Sust. Urb. Dev., SPbWOSCE Russian Federation, vol. 170, no. 02007, June 2018. DOI: $10.1051 /$ matecconf/201817002007.

[8] G.Yu. Silkina, "Information and communication technologies in ensuring of innovative development," Pr. of the 29th Int. Bus. Inf. Man. Ass. Conf. - Educ. Exc. and Inn. Man. Thr. Vis. 2020: From Reg.Dev. Sust. to Gl. Ec. Gr. Austria, vol. 1, pp. 1165-1176, May 2017.

[9] L. Lang, A. Mohnen, "An organizational view on transport transitions involving new mobility concepts and changing customer behavior," Environmental Innovation and Societal Transitions, 2019, vol. 31, pp. 54-63.

[10] J. Bulteau, T. Feuillet, S. Dantan, "Carpooling and carsharing for commuting in the Paris region: A comprehensive exploration of the individual and contextual correlates of their uses," Travel Behaviour and Society, 2019, vol. 16, pp. 77-87, July 2019.

[11] I. Zapivakhin, I. Ilin, A. Levina, "Public private partnership as city project management technology," MATEC Web of Conf. Int. Sc. Conf. on Bus. Tech. for Sust. Urb. Dev., SPbWOSCE Russian Federation, vol. 170, no. 01037, June 2018. DOI: 10.1051/matecconf/201817001037

[12] M. Gohar, M. Muzammal, A. Ur Rahman, "SMART TSS: Defining transportation system behavior using big data analytics in smart cities," Sustainable Cities and Society, 2018, vol. 41, pp. 114-119.

[13] O.Y. Iliashenko, A.I. Levina, A. Dubgorn, "Using business intelligence technologies for evaluation of road network organization," Pr. of the 30th Int. Bus. Inf. Man. Ass. Conf., IBIMA 2017 - Vision 2020: Sust. Ec. Dev., Inn. Man., and Gl. Gr., Spain, vol. 2017-January, pp. 2144-2155, November 2017.

[14] H. Elleuch, J. Rouis, "Devising a smart control and urban management network based on determination of the obstruction points of road traffic in Sfax," SM2C 2017 Int. Conf. on Sm., Mon. and Cont. Cit. Tunisia, no. 8071830, pp. 111-116, February 2017.

[15] J. Sasi Bhanu, J.K.R. Sastry, "On building cognitive expert system for managing road traffic within smart cities," Ponte, 2017, vol. 73(7), pp. 98-109.

[16] I.V. Ilin, O.Y. Iliashenko, A.I. Klimin, K.M. Makov, "Big data processing in Russian transport industry," Pr. of the 31st Int. Bus. Inf. Man. Ass. Conf., IBIMA 2018: Inn. Man. and Ed. Exc. Thr. Vis. 2020, Italy, pp. 1967-1971, April 2018.

[17] A. Lakštutienè, "Correlation of the indicators of the financial system and gross domestic product in European Union countries. In?inerin? ekonomika," 2008, vol. 3, no. 58, p. 7-18.

[18] N. Mehra, "Impact of foreign direct investment on employment and gross domestic product in India," International Journal of Economics and Research, 2013, vol. 4, no. 4, pp. 29-38.

[19] S. Hooda, 2011. "Study of FDI and Indian economy," [Online] pp. 114-115. Available at: http://www.nitkkr.ac.in/Sapna_Hooda_Thesis_A_Study_of_FDI_and _Indian_Econo my.pdf [Accessed 19 February 2012].

[20] P.L. Cox, B.A. Friedman, T. Tribunella, "Relationships among Cultural Dimensions, National Gross Domestic Product, and Environmental Sustainability," Journal of Applied Business and Economics, 2011, vol. 12(6), pp. 46-56.

[21] C. Qiang, C. Rossotto, 2009, "Economic Impacts of Broadband, (Information and Communications for Development: Extending Reach and Increasing Impact," Washington, DC, World Bank, pp. 3550 .

[22] N. Czernich, O. Falck, T. Kretschmer, L. Woessman, "Broadband infrastructure and economic growth," Economic Journal, 2011, vol. 121, no. 552, pp. 505-553.

[23] H. Varian, R. Litan, A. Elder, J. Shutter, "The Net Impact Study," 2002.

[24] E. Brynjolfsson, L.M. Hitt, "Computing productivity: firm-level evidence," Review of Economics and Statistics, 2003, vol. 85, no. 4, pp. 793-808. DOI: 10.1162/3465303772815736.

[25] R.L. Katz, P. Koutroumpis, "Measuring digitization: A growth and welfare multiplier," Technovation, 2013, vol. 33, no. 10-11, pp. 314319. DOI 10.1016/j.technovation.2013.06.004 
[26] S. Kalyazina, A. Borremans, A. Dubgorn, "Participation of citizens in sustainable development of big cities," (2018) MATEC Web of Conferences, Int. Sc. Conf. Env. Sc. for Constr. Ind., ESCI 2018, Viet Nam, vol. 193, no. 01029, August 2018. DOI: 10.1051/matecconf/201819301029.
[27] T. Kharlamova, "Monitoring as an instrument of Sustainable Urban Development," MATEC Web of Conf. Int. Sc. Conf. on Bus. Tech. for Sust. Urb. Dev., SPbWOSCE Russian Federation, vol. 170, no. 02009, June 2018. DOI: 10.1051/matecconf/201817002009.

[28] EasyParkGroup, "Report Smart Cities Index 2019," unpublished. 